\title{
Lacrimal gland cyst: Computed tomographic appearance
}

\author{
ROBIN E. OSBORN, D.O. \\ San Diego, California \\ CARL M. FISHER, D.O. \\ Tulsa, Oklahoma \\ SAEID MOJTAHEDI, M.D. \\ Chicago, Illinois
}

\begin{abstract}
A cyst of the lacrimal gland is a rare cause of a lateral orbital mass which arises from ducts within the gland. They usually involve the palpebral lobe of the lacrimal gland and project into the superotemporal cul-desac. They present clinically as a bluish, cystic-appearing mass which often transilluminates. They may cause pain or abnormal tearing, but they usually do not cause proptosis. Management is surgical. Radiographic evaluation with computed tomography demonstrates a sharply marginated, nonenhancing, cystic mass anterior to the lacrimal gland which projects into the lateral orbital cul-de-sac.
\end{abstract}

A cyst involving the lacrimal gland is rare ${ }^{1-5}$ The incidence of this finding is not known. A review of the literature yielded few computed tomographic (CT) descriptions of a lacrimal gland cyst. ${ }^{3-5}$ We present the typical CT appearance and discuss the differential diagnostic considerations and management of lacrimal gland cysts.

\section{Case report}

A 50-year-old man presented with an intermittently painful left eye of 1 week's duration. There was no history of trauma, infection, or inflammation involving the eye. The initial clinical evaluation demonstrated an approximately 1-cm., dark, hemorrhagic-appearing mass involving the superotemporal cul-de-sac of the left eye. The mass had become noticeable one week earlier and was initially pink in color. There was no epiphora, ker- atitis sicca, diplopia, or proptosis.

CT of the orbits demonstrated a low-density, smoothly marginated, $1-\mathrm{cm}$. mass anterior to the left lacrimal gland (Fig. 1). The mass projected into the superotemporal cul-de-sac. There was no enhancement after intravenous contrast infusion, bony erosion, or destruction.

The cyst was completely excised via a transconjunctival approach with sparing of the lacrimal gland. Pathologic examination of the surgical specimen revealed a cyst lined by a single layer of cuboidal epithelium which merged with a normal lacrimal duct. The diagnosis of a cyst of ductal origin was made on the basis of this observation.

\section{Discussion}

Epithelial cysts of the lacrimal gland arise from dilatation of a lacrimal duct and most commonly involve the palpebral lobe. ${ }^{1}$ They are believed to occur after infection, inflammation, or trauma to the lacrimal gland. Clinically, these cysts present as a superotemporal cul-de-sac mass of variable duration from weeks to years. There may also be associated eye pain, keratitis sicca, epiphora, and proptosis. Grossly, they are usually bluish in color, appear cystic, and are transilluminable. ${ }^{2-5}$ The cyst may become hemorrhagic, ${ }^{2}$ as in the present case.

The treatment for lacrimal gland cyst is complete resection. The surgical method utilizes a transconjunctival approach where the goal is to spare as much lacrimal tissue as possible. ${ }^{4,5}$ These cysts may recur and contralateral involvement has been noted ${ }^{5}$

Histologically, a lacrimal gland cyst wall may be composed of lamellated collagen lined by squamous or columnar pseudostratified nonkeratinized epithelial cells, ${ }^{4}$ a single layer of epithelium, or a 


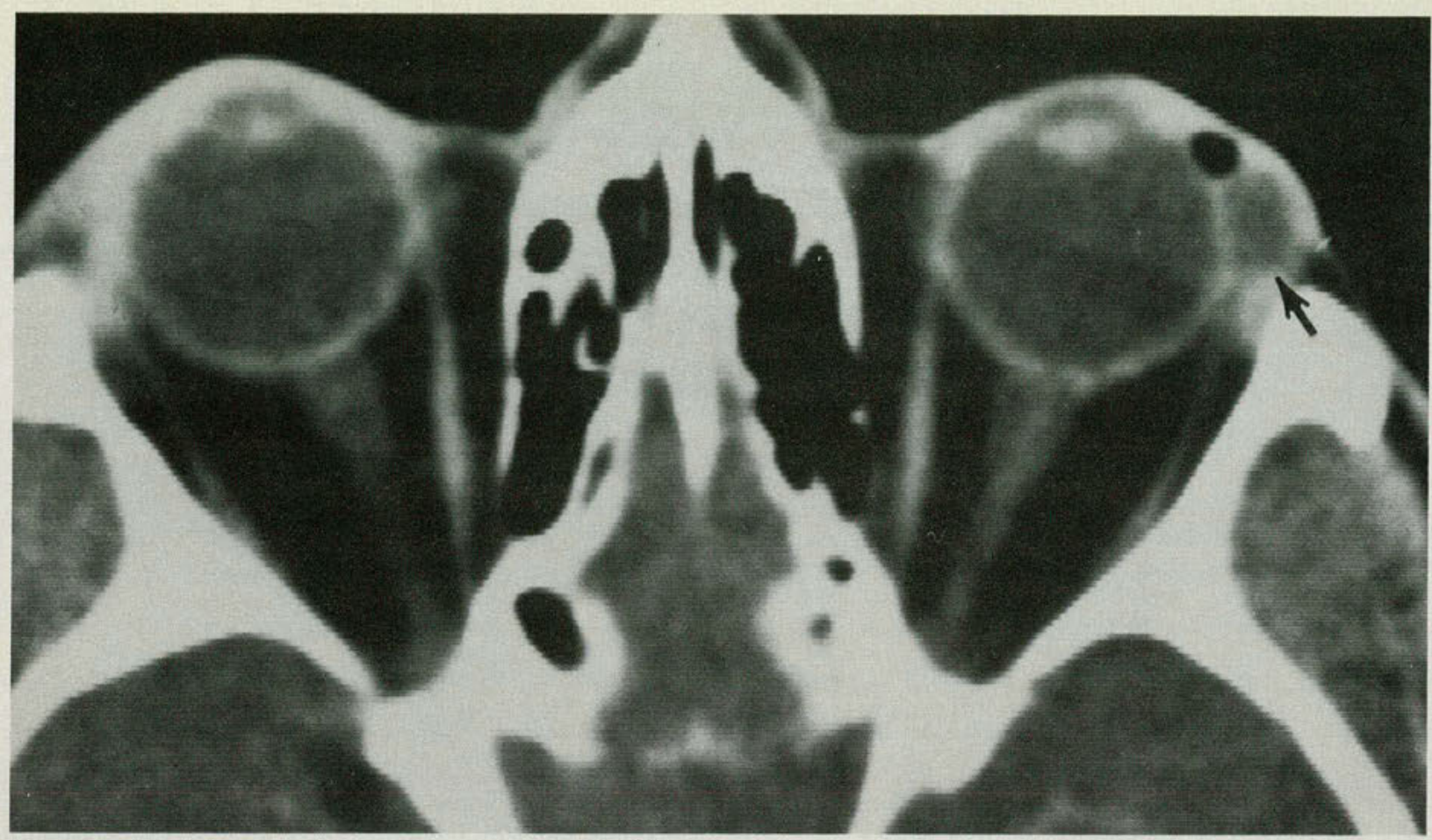

Fig. 1. Lacrimal gland cyst. The cyst lies anterior to the left lacrimal gland and bulges into the lateral fornix (arrow).

double layer of epithelium composed of an inner cuboidal and outer myoepithelial lining. This latter configuration is most typical of a lacrimal ductal cyst. ${ }^{3}$ Lacrimal tissue may be incorporated into the cyst wall. ${ }^{5}$

Cysts of the lacrimal gland must be differentiated from other lacrimal fossa region masses. ${ }^{2}$ Smith and Rootman ${ }^{3}$ encountered a series of other lacrimal region cysts which included dermoid cysts, laterally located frontal sinus mucoceles, implantation cysts, aneurysmal bone cysts, and a lateral rectus muscle cyst. Parasitic and conjunctival cysts are other cystic lesions to be considered as well. 1,3

Cysts arising from areas other than the palpebral lobe of the lacrimal gland are rare. Cysts may arise from the orbital lobe of the lacrimal gland, accessory lacrimal glands of Krause and Wolfring, or from ectopic lacrimal tissue which may involve any part of the orbit. ${ }^{2}$

The CT appearance of a lacrimal gland cyst consists of a low density, well-marginated, nonenhancing, cystic lesion lying adjacent the lacrimal gland. ${ }^{4,5}$ The cyst was of soft tissue density in one of the three cases illustrated in the literature. ${ }^{3}$ The cyst usually bulges into the lateral fornix or cul-desac of the orbit. ${ }^{3-5}$ The CT scan may demonstrate proptosis. $^{3}$

CT may not always differentiate benign from malignant or inflammatory from neoplastic condi- tions. ${ }^{6}$ It will usually delineate cystic components which may be present allowing differentiation from solid masses such as primary and secondary lacrimal region neoplasms. ${ }^{4} \mathrm{CT}$ may not differentiate lacrimal gland cyst from any other cystic mass involving the lacrimal fossa.

Bony erosion or expansion is not a finding associated with lacrimal gland cyst. Bony changes may be found with dermoid cyst and other primary and secondary lacrimal gland region neoplasms. The presence of fat within a lacrimal region mass is compatible with dermoid cyst. Rarely will a primary lacrimal gland tumor, i.e. pleomorphic adenoma, contain cystic spaces and mimic an epithelial cyst. ${ }^{7}$ Associated findings such as bony changes, contrast enhancement, and relationship to the lacrimal gland may assist in discovering the true nature of the lesion in such a case.

\section{Summary}

A lacrimal gland cyst is a rare, benign cyst which usually involves the palpebral lobe of the lacrimal gland. They are felt to result from antecedent trauma, infection, or inflammation of the lacrimal gland. They present as a mass protruding into the lateral fornix of the orbit from the lacrimal fossa region. They are diagnosed clinically. The CT scan findings should suggest the correct diagnosis, although other cystic lesions cannot be excluded. These lesions are treated by complete surgical 


\section{transconjunctival removal.}

1. Duke-Elder, S.: The ocular adenexa. Textbook of ophthalmology. C.V. Mosby Co., St. Louis, pp. 5201-5368, 1952

2. Bullock, J.D., et al.: Lacrimal ductal cysts. Ophthalmology 93:1355-60, Oct 86

3. Smith, S. and Rootman, J.: Lacrimal ductal cysts. Presentation and management. Surv Ophthalmol 30:245-50, Jan-Feb 86

4. Hornblass, A. and Herschorn, B.J.: Lacrimal gland duct cysts. Ophthalmic Surg 16:301-6, May 85

5. Brownstein, S., et al.: Orbital dacryops. Ophthalmology 91:1424-8, Nov 84

6. Balchunas, W.R., Quencer, R.M., and Byrne, S.F.: Lacrimal gland and fossa masses. Evaluation by computed tomography and A-mode echography. Radiology 149:751-8, Dec 83
7. Peyster, R.G. and Hoover, E.D.: Primary orbital neoplasma. In Computerized tomography in orbital disease and neuro-ophthalmology. Year Book Medical Publishers, Inc., Chicago, pp. 21-56, 1984

Dr. Osborn is an attending neuroradiologist, Department of Radiology, Section of Special Studies, Naval Hospital, San Diego, California. Dr. Fisher is an attending ophthalmologist, Oklahoma Osteopathic Hospital, Department of Ophthalmology, Tulsa, Oklahoma. Dr. Mojtahedi is director of neuroradiology, Department of Radiology, University of Chicago Medical Center, Chicago, Illinois.

Dr. Osborn, Department of Radiology, Section of Special Studies, Naval Hospital, San Diego, California 92134. 